\title{
Measurements of EEG Alpha Peak Frequencies Over the Lifespan: Validating Target Ranges on an In-Clinic Platform
}

\author{
David Joffe ${ }^{a}$, D S Oakley ${ }^{a}$ F. Arese Lucinia and F. X. Palermo ${ }^{b}$ \\ ${ }^{a}$ WAVi Research, Boulder CO ${ }^{b}$ University of Colorado School of Medicine, Aurora, CO
}

\begin{abstract}
Background: Peak individual alpha frequencies (IAF) extracted from an EEG exam can provide novel sources of information regarding brain function. This information can help measure and track changes in cognition arising from conditions such as concussion or unhealthy aging.
\end{abstract}

Objective 1: To validate a method for combining eyes-closed EEG with eyes-closed audio P300 ERP in order to streamline testing times involved in IAF extraction.

Objective 2: To validate age-stratified target ranges of IAF collected in clinic against published research.

Objective 3: To validate the stability of IAF for data collected in routine clinical settings.

Participants: Two thousand twenty-five subjects aged 13-90.

Methods: EEG with audio P300 was collected as part of a health screening exam for studies through Colorado University, Children's Hospital Colorado, Boone Heart Institute, WAVi Co., and various clinics alongside other clinical evaluations.

Results: (1) No differences were seen between IAF extracted during an eyes-closed resting and the P300 protocol. (2) The age-related AF trends measured in clinic match the age-related trends from previous research. (3) IAF remained stable over the course of 0-2 years in a test-retest dataset.

Conclusion: In-clinic measures of peak EEG frequency corroborate the age-related trends of published research taken over the last several decades and IAF. These results also confirm that IAF is a stable trait, making it useful for within-person longitudinal tracking. By following changes in IAF over time, deviations from normal CNS functioning, such as onset or progression of disease, can be monitored.

\section{Keywords}

Electroencephalogram (EEG), Alpha Power, Alpha Frequency, Alpha Peak Frequency (AF), Peak Individual Alpha Frequency (IAF), P300, Event Related Potential (ERP), Brainwave, Amplitude, Trail Making Test, Physical Reaction Time. 


\section{Introduction}

The alpha frequency, generally between 8 and $12 \mathrm{~Hz}$, is the dominant frequency of the human electroencephalogram (EEG), ${ }^{12}$ as shown in Figure 1. Measured during relaxed wakefulness, the power of the alpha rhythm is typically strongest in the eyes-closed condition. The peak of the alpha frequency (AF) can provide information on central nervous system functioning as well as the status of mental health and cognitive functioning. Significant correlations between AF and a large variety of cognitive measures have been observed, ${ }^{34567}$ perhaps suggesting links between AF and speed of information processing, the timing of neural inhibition, or the gating of information in the brain. 891011

Clear changes in the alpha rhythm are seen as the human brain ages, including AF slowing, reduction in alpha power, and a shift in the posterior-to-anterior direction. 51213141516171819202122 Slowing of the EEG has also been found to indicate CNS pathology, in particular slowing of AF has been observed repeatedly in patients with dementia. 232425262728293031323334

AF alone, however, may be of limited diagnostic value because the majority of these studies focus on comparing groups of individuals with respect to group differences and this may not always translate to identification of CNS pathology at the individual level. For example, even if the mean AF of a group with the diagnosis of dementia is found to be slower than the mean AF of healthy controls, an individual may still be located within the distribution of healthy adults.

An individual's peak alpha frequency (IAF), however, is considered a stable marker of a neurophysiological trait and can therefore provide longitudinal information on a patient. ${ }^{35} 363738394041$ Even when large gains in cognitive performance are achieved, the marker remains stable. ${ }^{42}$ Accounting for some of this stability is the observation that features of the alpha rhythm are likely to have a strong genetic component. ${ }^{43} 444546474849$

Given the high stability in the absence of neuropathological processes, IAF may be a valuable marker for monitoring neuropathological changes within individuals. Where single tests may possess poor sensitivity and/or specificity, by following within-person changes over time IAF can be valuable for monitoring deviations from normal CNS functioning, such as the onset or progression of disease. ${ }^{42}$ For this reason, IAF may be most valuable in tracking healthy individuals and looking for early changes before perhaps even symptoms arise.

For such monitoring to occur, this well-studied metric needs to move from research settings into standard clinical practice, including standard wellness visits. The objective here is to compare a large AF dataset 
collected in clinic to published research. In particular, we want to validate a clinical method involving a low-cost system designed to maximize information and minimize testing times. This includes concurrent testing of eyes-closed resting EEG protocols with eyes-closed EEG evoked response protocols. ${ }^{50}$ To that end the objective of this paper is to (1) validate the use of eyes-closed P300 to collect eyes-closed resting IAF measures and (2) validate data collected in routine clinical settings against published trends of AF over the lifespan (3) validate the within-person stability of IAF from an in-clinic sample.

\section{Methods}

\section{Subjects}

While the subjects for this study were comprised of 2025 subjects from previous or ongoing studies, it is not intended that they represent a normal control for a general population, rather it is intended to provide a target reference. One of the goals of this study is to compare in--clinic data with historical research to test the validity of large-scale screening. This study includes 3 control groups (13-16 years of age, 1723 , and 81-90) which will anchor the resulting age-matched curves as discussed below. It may be the case that these controls perform differently from a found in a normal population, where 2 of these control groups were taken from elite club, High School, or NCAA athletic teams while those in the oldest age range were volunteers living independently, still interested in brain science, and still interested in their brain performance. Each group will be discussed individually, but because of the suspected other-than-normal performance, we will focus on age trends and refer to this reference group as a target reference, with end points as discussed, rather than a normal reference. It is important to note that male/female differences are not the focus of this paper which is to compare trends to literature to establish a reference target.

All studies were approved by appropriate IRB's and written informed consent was obtained from the participants before study intake.

\section{Ages 8-12}

73 subjects aged 8-12 were taken from three previous studies: a study that followed athletes over the course of their sports seasons in Texas and Washington, control subjects measured as part a beta test to explore the outcome of an educational/wellbeing intervention program in a school representing low-income students, ${ }^{51}$ and minors accompanying WAVi study volunteers discussed below. Of these, 57 were accepted as per the artifacting criteria discussed below. 
This control group comprises 94 subjects from a previous study following athletes aged 13-16 over the course of their sports seasons and at 4 different sites. These subjects are participants in youth soccer and youth basketball representing all players from single teams. To follow the objectives of this study (as well as the above-mentioned studies) which involves real clinical settings, our exclusion criteria are minimal. Therefore the only exclusions are players who had lower than $80 \%$ yield as per the artifacting criteria outlined elsewhere. ${ }^{52}$ Of these subjects, 75 were deemed to have sufficient yield and 66 returned and completed a valid post-season second test which will be used to discuss test-retest variability.

\section{Ages 17-23}

This second control group is taken from a previous study that followed 364 athletes aged 17-23 over the course of up to 4 sports seasons and at 5 different sites. These subjects are participants in NCAA Div. 1 men's football (172 players, representing all players from a single team), woman's soccer (29 NCAA Div. 1 , representing all players from a single team), men's high school football (142 players, representing all seniors from a single team), and semipro men's ice hockey (20 players, representing all players from a single team). ${ }^{9}$

In these previous studies, these subjects were controls against which pre-contact, post-concussion and return-to-play groups could be compared. To follow the objectives of this study (as well as the abovementioned studies) which involves real clinical settings, and because the primary marker being studied is nonspecific, our exclusion criteria are minimal. The "control" group, therefore, is a reference group taken from all players participating on these teams and exclusions are limited to the players who fell asleep during the first-year test and passing the artifact criteria discussed below, leaving a total of 313 players comprising the baseline reference group of Table I. Of these subjects, 70 returned injury free to completed a valid second test, which will be used to discuss test-retest variability.

\section{Ages 24-30}

138 assessments were included for individuals aged 24-30 who were measured in clinic at baseline, where some were to be tracked over the course of various interventions. Subjects include patients who visited the Boone Heart Institute Colorado for a combined preventative cardiology and EEG/ERP evaluation from June 2014 through June 2017. Only first-time patients receiving an initial evaluation were included in the sample, which was also used for a preventative cardiology study. ${ }^{6}$ Because this is a target reference study, the exclusion criteria are minimal, the criteria being those who were taking beta-blockers or psychiatric medication and those who had lower than $80 \%$ yield on evoked responses due to artifact. 
Also included were subjects from Natural Bio Health (NBH) Texas for a first-time preventative wellness exam, evaluated from 2017 through 2018; and a random sample of subjects measured for demonstration purposes at 5 medical conferences.

The remaining subjects were volunteers who were known to or associated with the study team and wanted to become pro-active in their brain health. In general, these reference subjects were well educated and wanted to use WAVi to compare pre-intervention to post-interventions where interventions typically included some form of lifestyle change. To follow the objectives of this study, which involves real clinical settings our exclusion criteria are minimal and all volunteers in this age group were analyzed for the purposes of this study.

Ages 31-40

217 individuals aged 31-40 were tracked over the course of various interventions and the above-mentioned clinics, conferences, and volunteers.

Ages 41-50

325 individuals aged 31-40 were tracked over the course of various interventions and the above-mentioned clinics, conferences, and volunteers.

Ages 51-60

397 individuals aged 31-40 were tracked over the course of various interventions and the above-mentioned clinics, conferences, and volunteers.

Ages 61-70

249 individuals aged 31-40 were tracked over the course of various interventions and the above-mentioned clinics, conferences, and volunteers.

Ages 71-80

64 individuals aged 31-40 were tracked over the course of various interventions and the above-mentioned clinics, conferences, and volunteers.

Ages 81-90

Our third control group comprises 42 people taken as volunteers, discussed above. This group were living independently, had not been diagnosed with dementia, and were by definition a population who had experienced what could be called successful cognitive aging. They provide an end point against the 20year-old athletes for our target reference. Of these subjects, 26 were deemed to have sufficient yield to be included in the study.

Ages 24-85 Test-Retest Study Group

This group comprises 67 tests from 8 volunteers to study test-retest for various ERP and qEEG parameters 
during eyes-closed audio P300.

Ages 24-58 Default Network Study Group

This group comprises 108 total tests taken from 8 volunteer control subjects from the test-retest study above and from 22 subjects collected at a clinical site testing various conditions such as mood, attention, and for baseline wellness. These tests are of the reliability of audio P300 protocol as a default network by comparing the qEEG metrics extracted during the standard eyes-closed qEEG protocol to those extracted during the eyes-closed audio $\mathrm{P} 300$ protocol, including the theta/beta ratio, left-right alpha asymmetry, and alpha mean frequency in both a control and a clinical setting.

\section{EEG acquisition and preprocessing}

The WAVi Brain Assessment (WAVi Co., Boulder, CO USA) was used to record an electroencephalogram (EEG) at $250 \mathrm{~Hz}$ with the position of the electrodes following the International 10-20 system. Reference electrodes were clipped onto the earlobes. EEG eyes-closed resting parameters were recorded on each patient during a 4-min audio P300 and, for some subjects, during a 4-min eyesclosed resting protocol.

\section{EEG extraction and analysis}

WAVi 9.8.18 research software was used to extract and analyze the EEG data (the results of which are intended to create the target ranges for WAVi Scan 1.0- software). Frequency spectra were extracted from the eyes-closed and P300 EEG protocols using standard Fourier methods (Figure 1). This analysis included automatic artifact rejection that excludes noise from EEG data with higher than acceptable amplitudes while also excluding excessive band frequency activities in the standard EEG bands (Delta, Theta, Alpha, and Beta) on an individual channel basis. Files were also visually inspected to confirm proper noise extraction. In addition, when no clear alpha peaks were found, particularly when the delta and/or theta backgrounds were too large, a null result was given. While not a common occurrence, this null result is more common for frontal alpha than for occipital where alpha is generally more pronounced.

Finally, we extract AF for 3 regions of the scalp: Frontal (average frequency for F3, F4, and Fz); Central-Parietal (C-P, average frequency for C3, C4, Cz, P3, P4, and Pz); and Occipetal (average frequency for $\mathrm{O} 1$ and $\mathrm{O} 2$ ). 


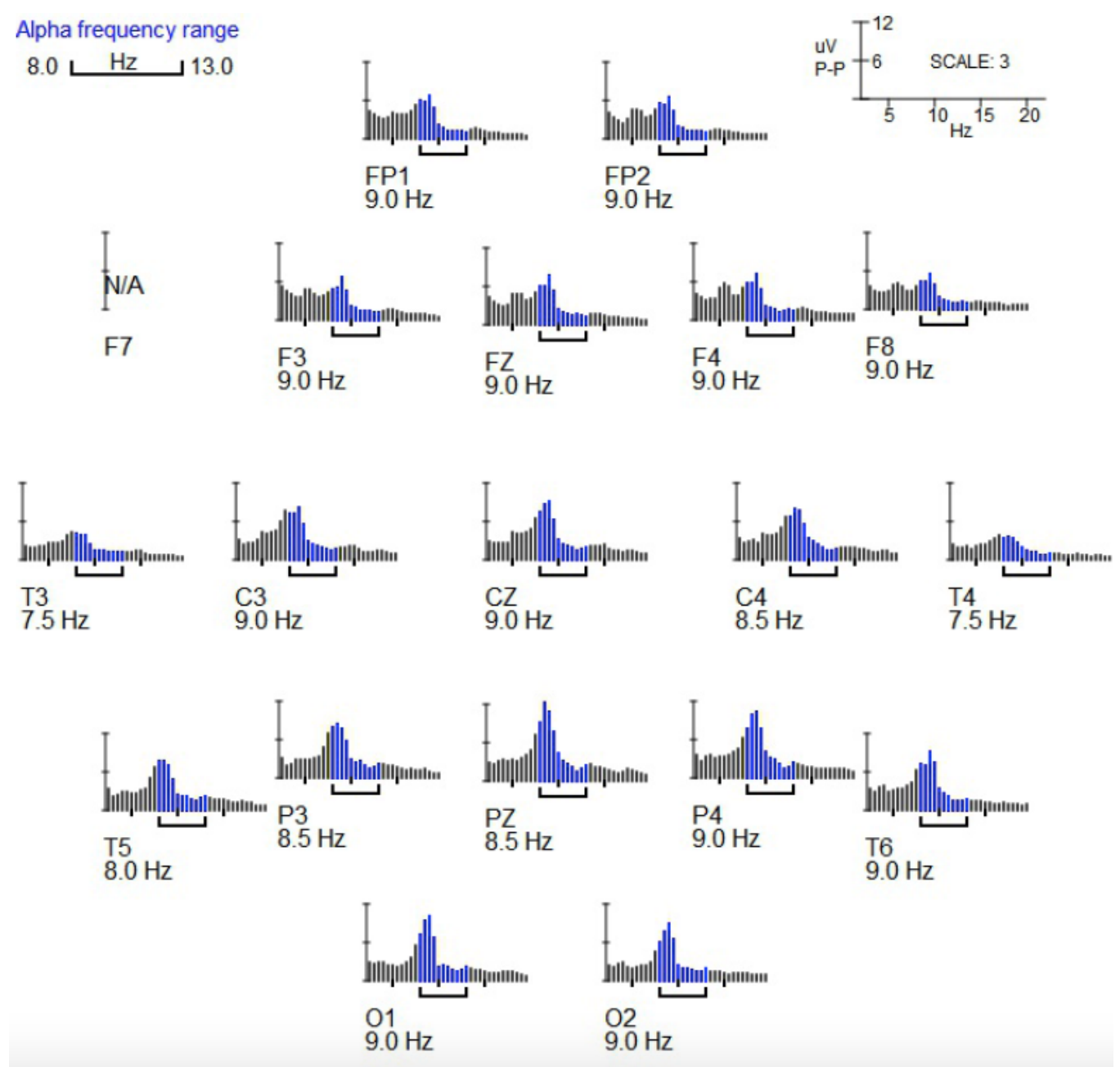

Figure 1. Frequency versus EEG amplitude spectra extracted from the WAVi platform for a sample subject, a healthy 25 -year-old control. Here the alpha window is displayed by the brackets below the spectra (set in this case from $8-13 \mathrm{~Hz}$ to guide the eye). Notice the strong alpha peak at all locations, with more theta contribution in the frontal areas for this patient.

\section{Results}

\section{AF During P300 and During Eyes-Closed Resting}

In order to maximize the information and minimize collection times, we compare IAF extracted during a 4-min eyes-closed Audio P300 (creating the AF of the P300 group) to those values extracted during standard eyes-closed resting (Table II). To minimize subject bias, the same 
number of eyes-closed and P300 tests were analyzed for each of the 30 subjects (22 subjects measured in clinic and 8 volunteer controls). From 108 reliable tests ( 80 in the front), no difference was seen between the two methods. Because we assume no difference in AF between these protocols, and to be consistent with the goal of validating a platform that maximizes information and minimizes testing times, the age-related IAF/AF trends of this study are extracted from this audio P300 protocol.

Table II AF extracted from IAF during standard eyes-closed resting and audio P300 protocols.
\begin{tabular}{|l|l|l|l|l|l|}
\hline Site & $\boldsymbol{A F}(\boldsymbol{S D})$ Eyes-Closed & $\boldsymbol{n}$ & $\boldsymbol{A F}(\boldsymbol{S D})$ P300 & $n$ & Difference Pvalue (CohenD) \\
\hline Frontal & $9.5(0.6) \mathrm{Hz}$ & 40 & $9.5(0.7) \mathrm{Hz}$ & 40 & $0.89(0.03)$ \\
\hline C-P & $9.6(0.9) \mathrm{Hz}$ & 52 & $9.4(1.0) \mathrm{Hz}$ & 52 & $0.29(0.21)$ \\
\hline Occipital & $9.7(1.0) \mathrm{Hz}$ & 54 & $9.7(0.8) \mathrm{Hz}$ & 54 & $0.79(0.05)$ \\
\hline
\end{tabular}

\section{AF Age Trends}

WAVi in-clinic data for AF over the lifespan, extracted during the eyes-closed P300 protocol, is shown in Figure 1. Strong agreement with previous studies in both magnitudes and age trends is seen. Figures 3-5 show AF over the lifespan for 3 locations of the scalp. As expected, the frequency declines with age but we also see a maturation effect with the fastest frequencies occurring at around 20-30 years of age. From this shape, the best fit for each scalp location is shown and from the resulting curve a target trendline can be established. Note high person-toperson variance where the "fastest" 85-year-olds are still faster than the "slowest" 20-year -olds, reinforcing the utility of IAF for tracking but not always for single tests. 
bioRxiv preprint doi: https://doi.org/10.1101/2021.10.06.463353; this version posted December 14,2021 . The copyright holder for this preprint (which was not certified by peer review) is the author/funder, who has granted bioRxiv a license to display the preprint in perpetuity. It is made available under aCC-BY-ND 4.0 International license.

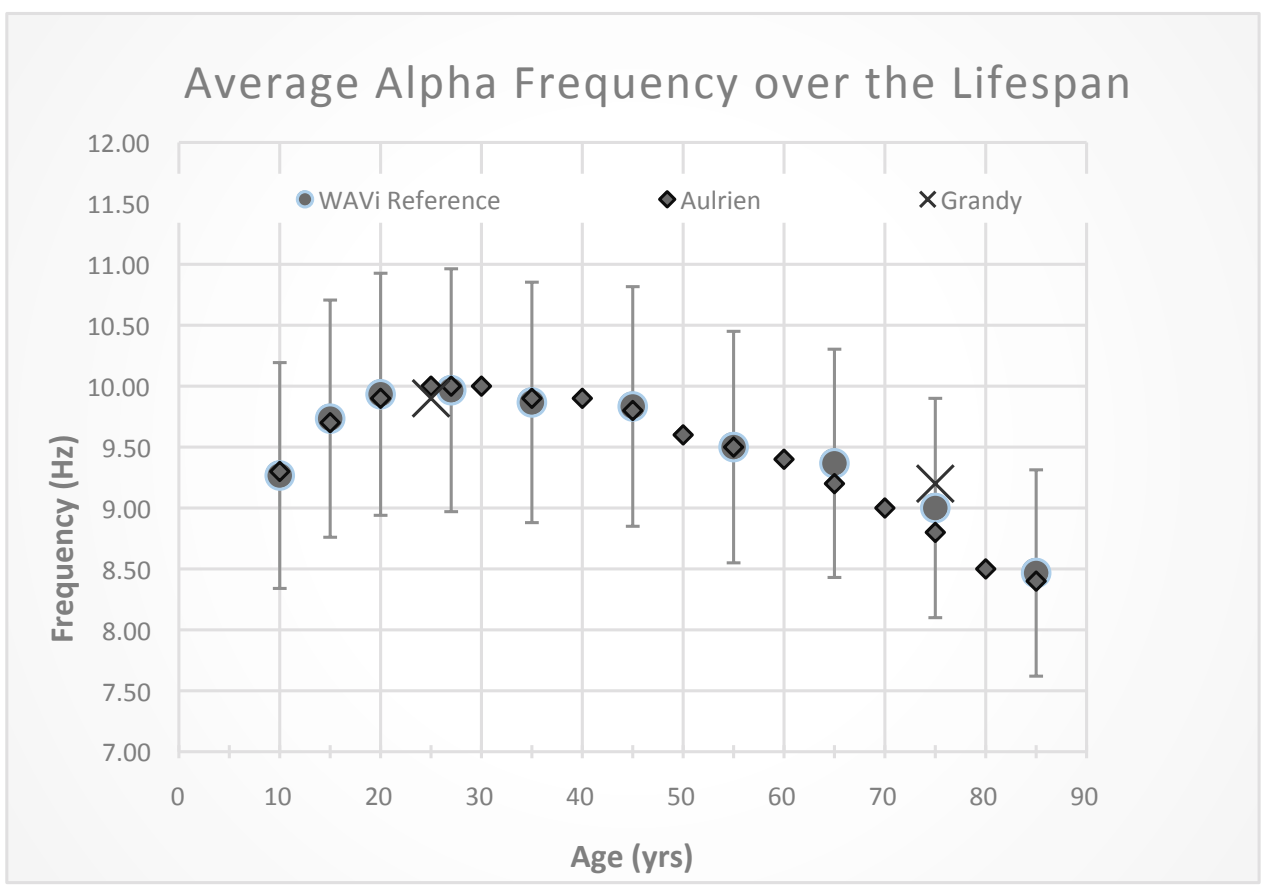

Figure 2. Comparison of Peak Alpha Frequencies extracted in routine clinical settings on the WAVi platform, as a function of age, to various studies. Here we take an average of frontal, central-parietal, and occipital locations.

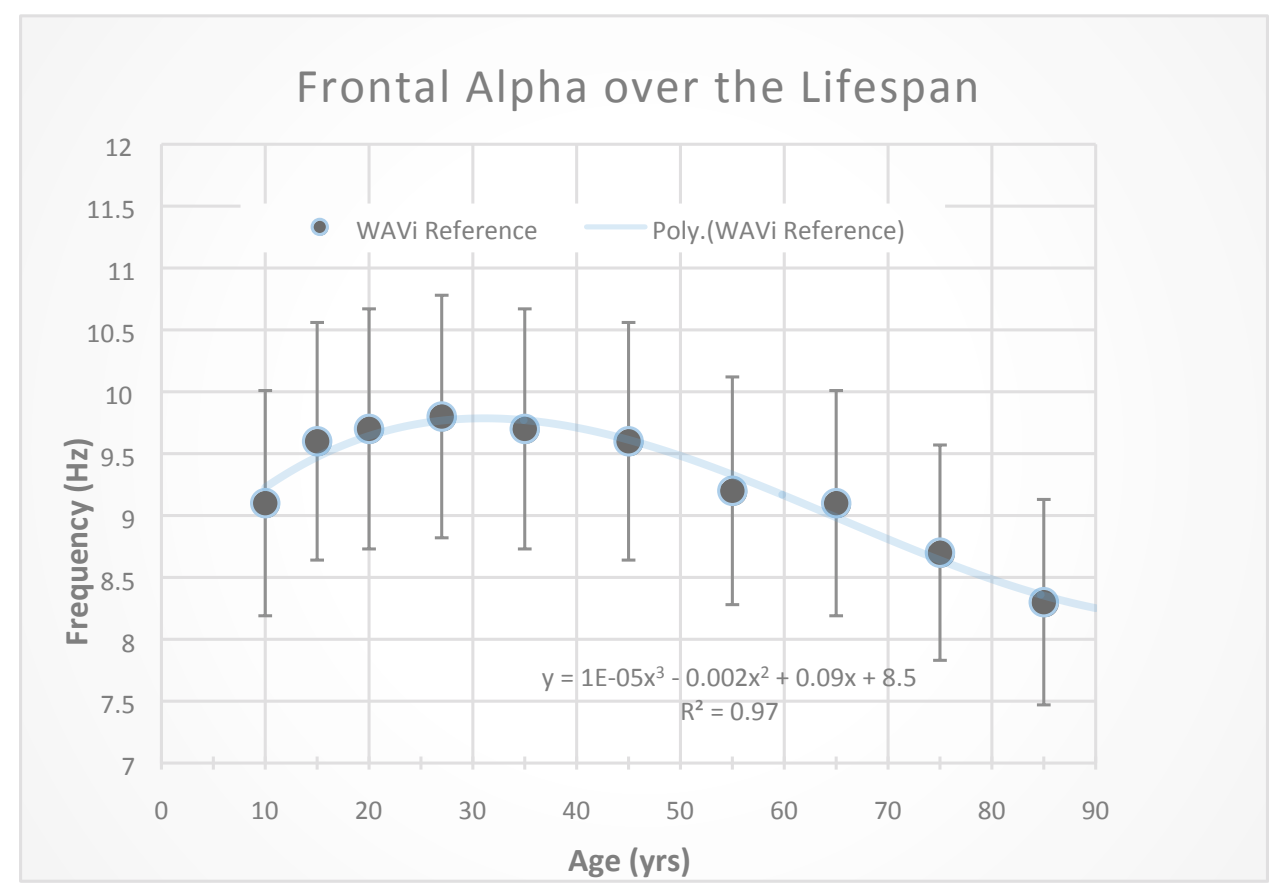

Figure 3. Peak Alpha Frequencies extracted in routine clinical settings on the WAVi platform, as a function of age, for the frontal locations. 
bioRxiv preprint doi: https://doi.org/10.1101/2021.10.06.463353; this version posted December 14,2021 . The copyright holder for this preprint (which was not certified by peer review) is the author/funder, who has granted bioRxiv a license to display the preprint in perpetuity. It is made available under aCC-BY-ND 4.0 International license.

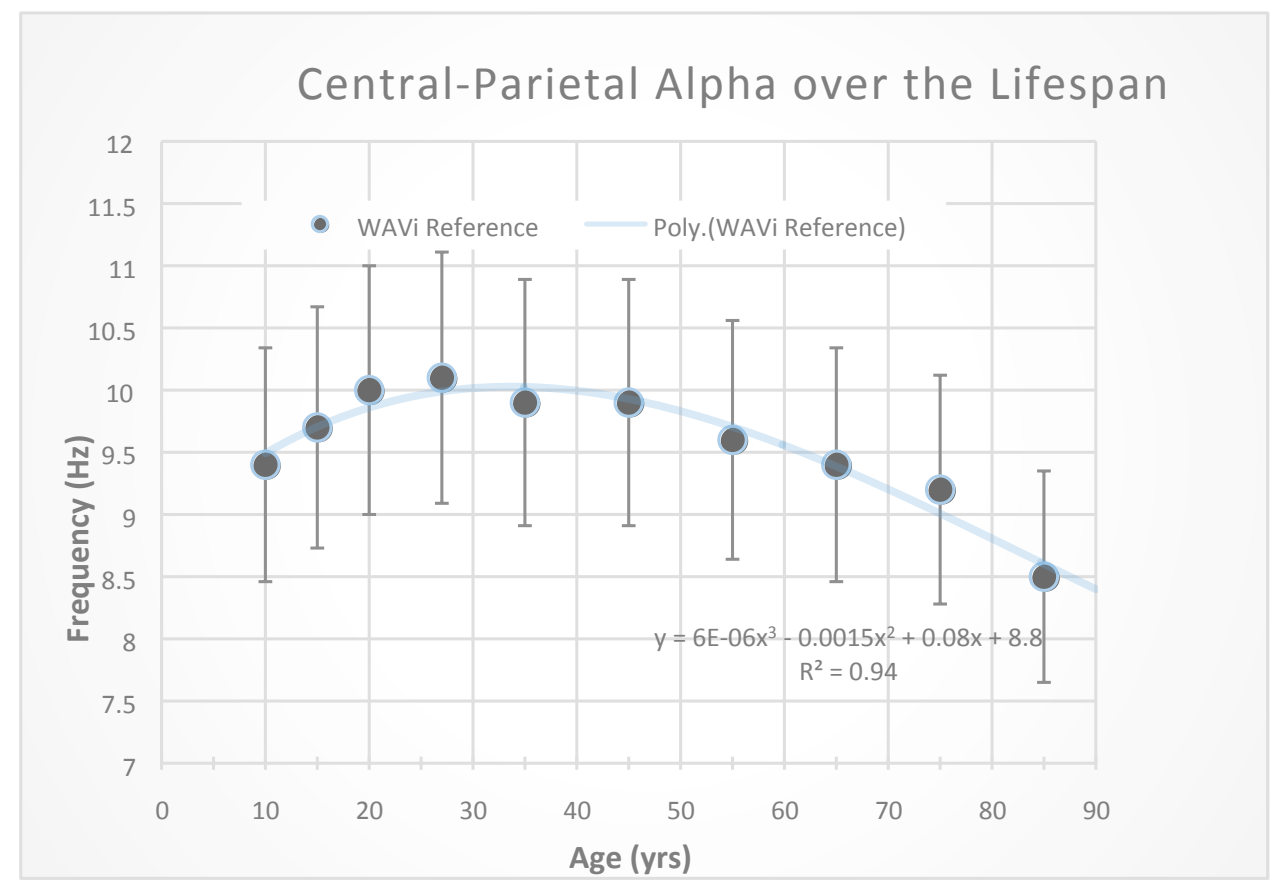

Figure 4. Peak Alpha Frequencies extracted in routine clinical settings on the WAVi platform, as a function of age, for the central-parietal locations.

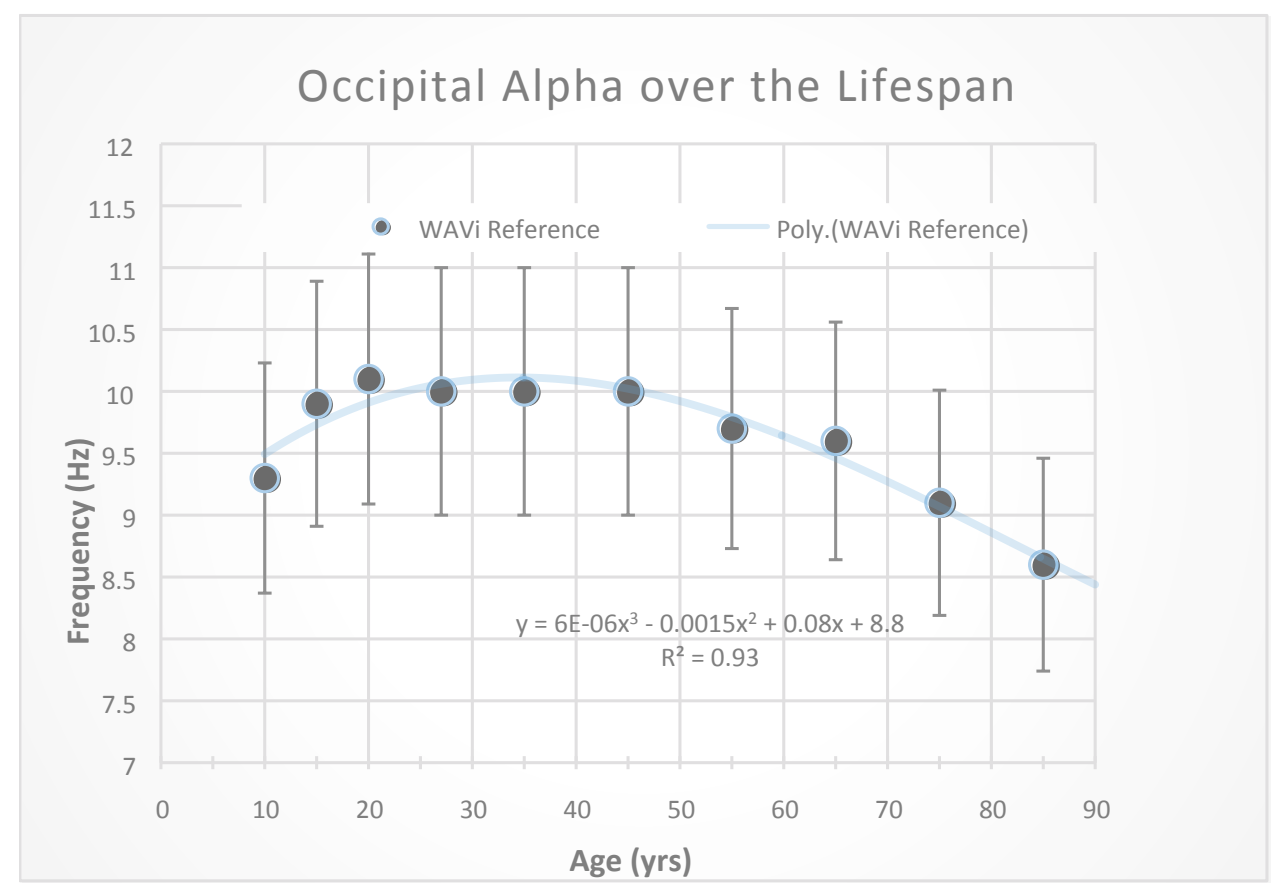

Figure 5. Peak Alpha Frequencies extracted in routine clinical settings on the WAVi platform, as a function of age, for the occipital locations. 
A note on variance: It is common practice in medicine to quote "normal ranges" (middle 68 percent of people in a Gaussian distribution) in order to provide context for both the clinician and client. To that end, we have found a variance of $+-10 \%$ provides a useful target range for all ages and regions.

\section{Test-Retest Variability}

IAF stability over time can be an important characteristic of intact general CNS functioning. While AF values alone may possess poor sensitivity and specificity as a clinical marker, IAF may constitute a robust and easy-to-assess metric for monitoring deviations from normal CNS functioning, such as progression of disease, by following within-person changes in IAF over time. ${ }^{42}$ As discussed, changes in IAF over time can be an early individual marker of pathology, but such use cases require that IAF remain stable within individuals in the absence of pathology.

Based on these previous studies, the expected intrapersonal variance in IAF from these data is stable. Table III shows test-retest results covering an age range of $24-85$ years where $84 \%$ of the subjects fell below +$0.2 \mathrm{~Hz}$ in change from their personal average over the course of 1 month to 2 years. These numbers provide a reference from which longitudinal changes can be studied, either after an event such as concussion, an intervention, or in order to monitor healthy aging.

Also shown in Table III are coefficients of variation $(\mathrm{CV})$, calculated as $100 * \mathrm{SD} /$ Average for each person and then averaged over each group. This provides context to compare IAF to other methods of physiologic assay, say for example HDL measures $(\mathrm{CV} \sim 24)$ and cholesterol $(\mathrm{CV} \sim 14) .{ }^{50}$ Table III CV values are lower than most other clinical assays, reinforcing the trait nature of these metrics and highlighting the clinical utility of longitudinal tracking. ${ }^{12}$

Table III Expected longitudinal change of IAF, with Coefficients of Variance (CV). Data were extracted over a 1-2 year period from the "test retest group" of Table I.

\begin{tabular}{|l|l|l|}
\hline Frontal $(\boldsymbol{C V})$ & $\boldsymbol{C}-\boldsymbol{P}(\boldsymbol{C V})$ & Occipital $(\boldsymbol{C V})$ \\
\hline$+-0.2 \mathrm{~Hz}(3.8)$ & $+-0.2 \mathrm{~Hz}(2.5)$ & $+-0.2 \mathrm{~Hz}(3.2)$ \\
\hline
\end{tabular}

\section{Conclusion}

In-clinic measures of AF extracted during an eyes-closed auditory P300 protocol corroborate the agerelated trends of published research taken over the last several decades. These results also confirm that IAF is a stable trait, making it useful for within-person longitudinal tracking. Regarding IAF collected 
during P300, it is reasonable to hypothesize that the eyes-closed resting protocol may not be as stable due to lack of control of the state of the patient (i.e drowsiness, concentration, etc.) while the P300 protocol requires a constant cognitive engagement. By following changes in IAF over time, deviations from normal CNS functioning, such as onset or progression of disease, can be monitored.

\footnotetext{
${ }^{1}$ Aurlien, H., Gjerde, I. O., Aarseth, J. H., Eldoen, G., Karlsen, B., Skeidsvoll, H., \& Gilhus, N. E. (2004). EEG background activity described by a large computerized database. Clinical Neurophysiology, 115, 665-673. ${ }^{2}$ Chiang, A. K., Rennie, C. J., Robinson, P. A., van Albada, S. J., \& Kerr, C. C. (2011). Age trends and sex differences of alpha rhythms including split alpha peaks. Clinical Neurophysiology, 122, 1505-1517. ${ }^{3}$ Angelakis, E., Lubar, J. F., Stathopoulou, S., \& Kounios, J. (2004). Peak alpha frequency: An electroencephalographic measure of cognitive preparedness. Clinical Neurophysiology, 115, 887-897.
} ${ }^{4}$ Anokhin, A. P., \& Vogel, F. (1996). EEG alpha rhythm frequency and intelligence in normal adults. Intelligence, $23,1-14$.

${ }^{5}$ Clark, C. R., Veltmeyer, M. D., Hamilton, R. J., Simms, E., Paul, R., Hermens, D., . . Gordon, E. (2004). Spontaneous alpha peak frequency predicts working memory performance across the age span. Int. J. Psychophysiol., 53, 1-9.

${ }^{6}$ Klimesch, W., Schimke, H., Ladurner, G., \& Pfurtscheller, G. (1990). Alpha frequency and memory performance. Journal of Psychophysiology, 4, 381-390.

${ }^{7}$ Mundy-Castle, A. C. (1958). Electrophysiological correlates of intelligence. Journal of Personality, 26, 184-199.

${ }^{8}$ Klimesch, W., Doppelmayr, M., Schimke, H., \& Pachinger, T. (1996). Alpha frequency, reaction time, and the speed of processing information. Journal of Clinical Neurophysiology, 13, 511-518.

${ }^{9}$ Klimesch, W., Sauseng, P., \& Hanslmayr, S. (2007). EEG alpha oscillations: The inhibition-timing hypothesis. Brain Research Reviews, 53, 63-88.

${ }^{10}$ Mazaheri, A., \& Jensen, O. (2010). Rhythmic pulsing: Linking ongoing brain activity with evoked responses. Frontiers in Human Neuroscience, 4, 177.

${ }^{11}$ Jensen, O., \& Mazaheri, A. (2010). Shaping functional architecture by oscillatory alpha activity: Gating by inhibition. Frontiers in Human Neuroscience, 4, 186.

${ }^{12}$ Maria G. Knyazeva, Elham Barzegaran, Vladimir Y. Vildavski, Jean-François Demonet. (2018). Aging of human alpha rhythm. Neurobiology of Aging, 69, 261-273.

${ }^{13}$ Basar, E. (2012). A review of alpha activity in integrative brain function: fundamental physiology, sensory coding, cognition and pathology. Int. J. Psychophysiol., 86,1-24.

${ }^{14}$ Gaál, Z.A., Boha, R., Stam, C.J., Molnár, M. (2010). Age-dependent features of EEG reactivity: spectral, complexity, and network characteristics. Neurosci. Lett., 479, 79-84.

${ }^{15}$ Hubbard, O., Sunde, D., Goldensohn, E.S. (1976). The EEG in centenarians. Electroencephalogr. Clin. Neurophysiol., 40, 407-417.

${ }^{16}$ Lodder, S.S., van Putten, M.J. (2011). Automated EEG analysis: characterizing the posterior dominant rhythm. $J$. Neurosci. Methods, 200, 86-93.

${ }^{17}$ Markand, O.N. (1986). Electroencephalogram in dementia. Am. J. EEG Technol., 26, 3-17.

${ }^{18}$ Peltz, C.B., Kim, H.L., Kawas, C.H. (2010). Abnormal EEGs in cognitively and physically healthy oldest-old: findings from the 90. study. J. Clin. Neurophysiol., 27, 292-295.

${ }^{19}$ Vysata, O., Kukal, J., Prochazka, A., Pazdera, L., Valis, M. (2012). Age-related changes in the energy and spectral composition of EEG. Neurophysiology, 44, 63-67.

${ }^{20}$ Babiloni, C., Binetti, G., Cassarino, A., Dal Forno, G., Del Percio, C., Ferreri, F., Ferri, R., Frisoni, G., Galderisi, S., Hirata, K. (2006). Sources of cortical rhythms in adults during physiological aging: a multicentric EEG study. Hum. Brain Mapp., 27, 162-172.

${ }^{21}$ Niedermeyer, E. (1997). Alpha rhythms as physiological and abnormal phenomena. Int. J. Psychophysiol., 26, 3149.

${ }^{22}$ Rossini, P., Del Percio, C., Pasqualetti, P., Cassetta, E., Binetti, G., Dal Forno, G., Ferreri, F., Frisoni, G., Chiovenda, P., Miniussi, C. (2006). Conversion from mild cognitive impairment to Alzheimer's disease is predicted by sources and coherence of brain electroencephalography rhythms. Neuroscience, 143, 793-803. 
${ }^{23}$ Cantero, J. L., Atienza, M., Gomez-Herrero, G., Cruz-Vadell, A., Gil-Neciga, E., Rodriguez-Romero, R., .. . Garcia-Solis, D. (2009). Functional integrity of thalamocortical circuits differentiates normal aging from mild cognitive impairment. Human Brain Mapp., 30, 3944-3957.

${ }^{24}$ d'Onofrio, F., Salvia, S., Petretta, V., Bonavita, V., Rodriguez, G., \& Tedeschi, G. (1996). Quantified-EEG in normal aging and dementias. Acta Neurologica Scandinavica, 93, 336-345.

${ }^{25}$ Gawel, M., Zalewska, E., Szmidt-Salkowska, E., \& Kowalski, J. (2007). Does EEG (visual and quantitative) reflect mental impairment in subcortical vascular dementia? Journal of the Neurological Sciences, 257, 11-16.

${ }^{26}$ Jelic, V., Johansson, S. E., Almkvist, O., Shigeta, M., Julin, P., Nordberg, A., . . Wahlund, L. O. (2000).

Quantitative electroencephalography in mild cognitive impairment: Longitudinal changes and possible prediction of Alzheimer's disease. Neurobiology of Aging, 21, 533-540.

${ }^{27}$ Montez, T., Poil, S. S., Jones, B. F., Manshanden, I., Verbunt, J. P., van Dijk, B.W., . . Linkenkaer-Hansen, K. (2009). Altered temporal correlations in parietal alpha and prefrontal theta oscillations in early-stage Alzheimer disease. Proc. Nat. Acad. Sci. U.S.A., 106, 1614-1619.

${ }^{28}$ Moretti, D. V., Babiloni, C., Binetti, G., Cassetta, E., Dal Forno, G., Ferreric, F., . . Rossini, P. M. (2004).

Individual analysis of EEG frequency and band power in mild Alzheimer's disease. Clinical Neurophysiology, 115, 299-308.

${ }^{29}$ Babiloni, C., Frisoni, G. B., Pievani, M., Toscano, L., Del Percio, C., Geroldi, C., . . Rossini, P. M. (2008). White-matter vascular lesions correlate with alpha EEG sources in mild cognitive impairment. Neuropsychologia, 46, 1707-1720.

${ }^{30}$ Moretti, D. V., Miniussi, C., Frisoni, G., Zanetti, O., Binetti, G., Geroldi, C.,. . . Rossini, P. (2007). Vascular damage and EEG markers in subjects with mild cognitive impairment. Clinical Neurophysiology, 118, 1866-1876.

${ }^{31}$ Prichep, L. S. (2007). Quantitative EEG and electromagnetic brain imaging in aging and in the evolution of dementia. Annals of the New York Academy of Sciences, 1097, 156-167.

${ }^{32}$ Stomrud, E., Hansson, O., Minthon, L., Blennow, K., Rosen, I., \& Londos, E. (2010). Slowing of EEG correlates with CSF biomarkers and reduced cognitive speed in elderly with normal cognition over 4 years. Neurobiology of Aging, 31, 215-223.

${ }^{33}$ Szelies, B., Grond, M., Herholz, K., Kessler, J., Wullen, T., \& Heiss, W. D. (1992). Quantitative EEG mapping and PET in Alzheimer's disease. Journal of the Neurological Sciences, 110, 46-56.

${ }^{34}$ Szelies, B., Mielke, R., Kessler, J., \& Heiss, W. D. (1999). EEG power changes are related to regional cerebral glucose metabolism in vascular dementia. Clinical Neurophysiology, 110, 615-620.

${ }^{35}$ Deakin, J. F. W., \& Exley, K. A. (1979). Personality and male-female influences on the EEG alpha rhythm. Biological Psychology, 8, 285-290.

${ }^{36}$ Gasser, T., Bächer, P., \& Steinberg, H. (1985). Test-retest reliability of spectral parameters of the EEG. Electroencephalogr. Clin. Neurophysiol., 60, 312-319

${ }^{37}$ Kondacs, A., \& Szabó, M. (1999). Long-term intra-individual variability of the background EEG in normals. Clinical Neurophysiology, 110, 1708-1716.

${ }^{38}$ Salinsky, M. C., Oken, B. S., \& Morehead, L. (1991). Test-retest reliability in EEG frequency analysis. Electroencephalogr. Clin. Neurophysiol., 79, 382-392.

${ }^{39}$ Doppelmayr, M., Klimesch, W., Pachinger, T., \& Ripper, B. (1998). Individual differences in brain dynamics: Important implications for the calculation of event-related band power. Biological Cybernetics, 79, 49-57.

${ }^{40}$ Klimesch, W. (1996). Memory processes, brain oscillations and EEG synchronization. Int. J. Psychophysiol., 24, 61-100.

${ }^{41}$ Klimesch, W. (1997). EEG-alpha rhythms and memory processes. Int. J. Psychophysiol., 26, 319-340.

${ }^{42}$ Grandy, T.H., Werkle-Bergner, M., Chicherio, C., Schmiedek, F., Lövden, M., Lindenberger, U. (2013). Peak individual alpha frequency qualifies as a stable neurophysiological trait marker in healthy younger and older adults. Psychophysiology, 50, 570-582

${ }^{43}$ Anokhin, A. P., Müller, V., Lindenberger, U., Heath, A. C., \& Myers, E. (2006). Genetic influences on dynamic complexity of brain oscillations. Neuroscience Letters, 397, 93-98.

${ }^{44}$ Anokhin, A. P., van Baal, G. C., van Beijsterveldt, C. E., de Geus, E. J., Grant, J., \& Boomsma, D. I. (2001). Genetic correlation between the P300 event-related brain potential and the EEG power spectrum. Behavior Genetics, 31, 545-554.

${ }^{45}$ Smit, C. M., Wright, M. J., Hansell, N. K., Geffen, G. M., \& Martin, N. G. (2006). Genetic variation of individual alpha frequency (IAF) and alpha power in a large adolescent twin sample. Int. J. Psychophysiol., 61, 235-243. 
${ }^{46}$ Smit, D. J., Posthuma, D., Boomsma, D. I., Geus, E. J., Smit, D. J. A., \& Geus, E. J. C. (2005). Heritability of background EEG across the power spectrum. Psychophysiology, 42, 691-697

${ }^{47}$ van Beijsterveldt, C. E. M., \& Boomsma, D. I. (1994). Genetics of the human electroencephalogram (EEG) and event-related brain potentials (ERPs): A review. Human Genetics, 94, 319-330.

${ }^{48}$ van Beijsterveldt, C. E. M., \& van Baal, G. C. M. (2002). Twin and family studies of the human electroencephalogram: A review and a meta analysis. Biological Psychology, 61, 111-138.

${ }^{49}$ Vogel, F. (1970). The genetic basis of the normal human electroencephalogram (EEG). Humangenetik, 10, 91114.

${ }^{50}$ Polich, J., Herbst, K. (2000). P300 as a clinical assay: rationale, evaluation, and findings. Int. J. Psychophysiol., 38, 3-19.

${ }^{51}$ Maroney, D.I. (2020). The Imagine Project: Using expressive writing to help children overcome stress and trauma. Pediatric Nursing, 46(6), 300-302.

${ }^{52}$ Lindsay Elliott, Julie Wilson, Pamela Wilson, Ariel Kiyomi Lepon, Gregg Brueck, MS, Gerald Clayton. (2017). Quantitative Electroencephalography in the Evaluation of Concussive Head Injury in Adolescent Children: A Preliminary Analysis. Proceedings of the 2017 American Academy of Physical Medicine and Rehabilitation Annual Assembly, Denver Colo. 